Annals of Pure and Applied Mathematics

Vol. 17, No. 1, 2018, 137-146

ISSN: 2279-087X (P), 2279-0888(online)

Published on 22 May 2018

Annals of

www.researchmathsci.org

DOI: http://dx.doi.org/10.22457/apam.v17n1a16

Pure and Applied

Mathematics

\title{
A Study on Job Preferences Given in Job Change Decision of Startup Company Workers in Chennai Using Integrated Fuzzy Multi Criteria Decision Making Methodology
}

\author{
R. Sophia Porchelvi and B. Snekaa* \\ Department of Mathematics, A.D.M College for Women (Autonomous) \\ Nagapattinam-611001, Tamilnadu, India \\ *Corresponding author. E-mail: bsnekaa@gmail.com
}

Received 14 April 2018; accepted 20 May 2018

\begin{abstract}
This paper deals with the problem of job change difficulties of start-up company workers in Chennai. Most of the workers in start-up companies feel hangout due to dissatisfied salary and job nature. Many even get confused what job is suitable for them and are in an urge to prefer the right career immediately. The job preferences of workers are studied using hybrid approach as a methodology integrating fuzzy analytic hierarchy process (FAHP) and fuzzy multi-objective optimization ratio analysis (FMOORA). FAHP helps to determine the weight of the decision criteria and FMOORA to evaluate the alternatives.
\end{abstract}

Keywords: Fuzzy Analytic Hierarchy Process, Buckley's Geometric Mean Method, Fuzzy Multi-Objective Optimization Ratio Analysis, Integration of fuzzy AHP and fuzzy MOORA.

AMS Mathematics Subject Classification (2010): 20F10

\section{Introduction}

Fuzzy set theory was introduced by Zadeh in 1965[1]. Fuzzy sets and fuzzy logic theory and applications are given by Klir and Yan in 1995 [2]. A fuzzy extension of Saaty's priority theory was discussed by Van Laarhoven and Pedrycz in 1983 [11]. Decisionmaking in a Fuzzy environment Management Science was dealt by Bellman and Zadeh in 1970 [5]. Studied fuzzy multiple attribute decision making which was discussed by Chen, Hwang and Hwang in 1992 [6]. And multi-criteria decision making methods and fuzzy sets was given by Kahraman in 2008 [8]. Then studied the analytic hierarchy process which was discussed by Saaty in 1980 [9]. Buckley in 1985 discussed fuzzy hierarchical analysis [10]. In order to tackle the decision making problems Balezantis, Balezantis and Brauers in 2012, given the Multimoora a multi-objective decision making method for linguistic reasoning with an application to personnel selection [13] and studied the MOORA method and its application to privatization in a transition economy which was dealt by Brauers and Zavadskas in 2006 [14]. 


\section{R. Sophia Porchelvi and B. Snekaa}

Occupation is defined as the set of organized activities based on knowledge and skills provided by education carried out in order to make a living. Job choice is the decision of an individual about which occupation is the most suitable to him/her. Changing a job is a daunting decision for professionals in competitive world. If the individual's expectation does not meet with life realities poor performance is inevitable. By understanding the factors affecting their choice individual's asses job alternatives better. The mismatch between the expectations and experience on the job leads to dissatisfaction and poor work performance and turn to another job search.

Job change decision is defined as a multiple criteria MCDM problem. The decision process is essentially an unconscious one influenced by emotions and intuition but due to limitations in human cognition and influential mechanism shaped by emotions it fails to offer a comprehensive solution. Fuzzy multi criteria decision making comes in handy to identify sets of factors that explain one's job choice and determine his/her job preference and decide better alternative that is good match for all needs of the individual. Parallel to the developments of new methods, each method's features are studied and advanced by using two or more MCDM methods and creating hybrid methods. Such methods have gained popularity.

In this paper, the job preferences of workers are studied using hybrid approach as a methodology integrating Fuzzy Analytic Hierarchy Process and Fuzzy MOORA. FAHP helps determine the weight of the decision criteria and FMOORA evaluates alternatives. For the problem six alternatives and eight criteria have been determined.

\section{Preliminaries}

2.1. Trapezoidal fuzzy number $\tilde{A}$ is a normal fuzzy number represented by the quadruplet $(a, b, c, d)$ where $a \leq b \leq c \leq d$ are real numbers and its membership function $\mu_{\tilde{A}}: X \rightarrow[0,1]$ is given below

$$
\mu_{\tilde{A}}(x)=\left\{\begin{array}{c}
\frac{x-a}{b-a}, \text { if } a \leq x \leq b \\
1, \text { if } b \leq x \leq c \\
\frac{x-d}{c-d}, \text { if } c \leq x \leq d \\
0, \text { if else }
\end{array}\right.
$$

\subsection{Arithmetic operations on trapezoidal fuzzy number} Let $\tilde{A}=\left(a_{1}, a_{2}, a_{3}, a_{4}\right)$ and $\tilde{B}=\left(b_{1}, b_{2}, b_{3}, b_{4}\right)$

(a) Addition of the two trapezoidal numbers can be denoted as $\tilde{A}+\widetilde{B}$ and it is defined by

$$
\tilde{A}+\tilde{B}=\left(a_{1}+b_{1}, a_{2}+b_{2}, a_{3}+b_{3}, a_{4}+b_{4}\right)
$$

(b) Subtraction of the two trapezoidal numbers can be denoted as $\tilde{A}-\tilde{B}$ and it is defined by

$$
\tilde{A}-\tilde{B}=\left(a_{1}-b_{1}, a_{2}-b_{2}, a_{3}-b_{3}, a_{4}-b_{4}\right)
$$

(c) Multiplication of the two trapezoidal numbers can be denoted as $\tilde{A} \times \tilde{B}$ and it is defined by

$$
\tilde{A} \times \tilde{B}=\left(a_{1} \times b_{1}, a_{2} \times b_{2}, a_{3} \times b_{3}, a_{4} \times b_{4}\right)
$$

(d) Complement of a trapezoidal fuzzy number can be denoted by $\tilde{A}^{c}$ and it is defined by

$$
\tilde{A}^{c}=\left(1-a_{4}, 1-a_{3}, 1-a_{2}, 1-a_{1}\right) .
$$


A Study on Job Preferences Given in Job Change Decision of Startup Company Workers in Chennai Using Integrated Fuzzy Multi Criteria Decision Making Methodology

(e) Defuzzification is the process of converting fuzzy numbers obtained from fuzzy inference into crisp values. For a trapezoidal number parameterized by $\left(a_{1}, a_{2}, a_{3}, a_{4}\right)$, then the defuzzification value $t$ of the trapezoidal fuzzy number. It is calculated by

$$
t=\frac{a_{1}+a_{2}+a_{3}+a_{4}}{4}
$$

\subsection{Fuzzy analytic hierarchy process (FAHP)}

Fuzzy AHP has an impressive capability to replicate human thoughts to perception and has been used by many researches in the literature. The consistency ratio (CR) calculations are done using crisp values of the fuzzy numbers as suggested by Chen and Hung (2010). According to Saaty [7], consistency ratio of an evaluation matrix should not exceed the acceptable level of 0.1 .

$$
\begin{gathered}
\mathrm{CI}=(\lambda \max -\mathrm{n}) /(\mathrm{n}-1) \\
\mathrm{CR}=\mathrm{CI} / \mathrm{RC} .
\end{gathered}
$$

\subsection{Buckley's geometric mean method}

The geometric mean method was employed by Buckley (1985) [8] extending Saaty's [7] AHP method to incorporate fuzzy comparison ratios $\mathrm{a}_{\mathrm{ij}}$ and situation of using linguistic variables. It involves the following steps.

(a) Construction of hierarchy, (b) Pair-wise comparison of decision makers, (c) Aggregating fuzzy weights, (d) Defuzzification, (e) Calculating final weights.

\subsection{MOORA over other MCDM methods}

- Newest MCDM methods constructed already knowing weak aspects of the older measures.

- Less computational time, low setup time and has a good stable nature.

- Very simple with minimum mathematical calculations.

MOORA method was introduced by Brauers and Zavadskas (2006) [12], fuzzy MOORA is used here for evaluation and ranking the alternatives.

\section{Solving MCDM models by integrating fuzzy AHP and fuzzy MOORA}

A combination of FAHP and FMOORA methods are effective to researchers. FAHP method is appropriate for determining job criteria weights and FMOORA approach is proposed for evaluating and ranking job alternatives. The integration approach involves the following steps

- Construct pair wise comparison matrices for all the criteria.

- Use geometric mean technique to define the fuzzy geometric mean and fuzzy weights of each criterion by Buckley (1985)[8].

- The procedure of defuzzification is to locate the best non fuzzy performance value (BNP) using COA and obtain criteria weightage.

- Based on the expert opinions of the decision makers, develop the fuzzy decision matrix.

- Normalize the fuzzy decision matrix using vector normalization procedure.

- Determine the weighted normalized fuzzy decision matrix. 


\section{R. Sophia Porchelvi and B. Snekaa}

- Calculate the overall ratings of beneficial and non-beneficial criteria, the overall ratings of an alternative.

- Determine the overall performance index.

- Based on the descending values of overall performance index, rank the alternatives from the best to worst. The alternative with the highest overall performance index is the most favorable choice.

\section{Integrated methodology algorithm}

Step 1: Creating decision criteria and alternatives.

Step 2: Defining linguistic variables and fuzzy scale.

Step 3: Pair wise comparison matrix for criteria is created

where each $\tilde{\mathrm{a}}_{\mathrm{ij}}=1 / \tilde{\mathrm{a}}_{\mathrm{ji}}$.

$$
\tilde{A}=\left[\begin{array}{ccc}
1 & \cdots & \tilde{a}_{1 n} \\
\vdots & \ddots & \vdots \\
\tilde{a}_{n 1} & \cdots & 1
\end{array}\right]
$$

Step 4: Defuzzification for all comparison matrices. Consistency index is calculated

$$
\begin{gathered}
\mathrm{CI}=(\lambda \max -\mathrm{n}) /(\mathrm{n}-1) \\
\mathrm{CR}=\mathrm{CI} / \mathrm{RC}
\end{gathered}
$$

where $\mathrm{n}$ is number of criteria

CI should not exceed 0.1.

Step 5: Geometric mean for each criteria is calculated

$$
\tilde{r}_{i}=\left(\tilde{a}_{i 1} \odot \tilde{a}_{i 2} \odot \ldots \odot \widetilde{a}_{i n}\right)^{1 / n}
$$

Step 6: Criteria weightage is calculated with normalization process
$\tilde{\mathrm{W}}_{\mathrm{i}}=\tilde{\mathrm{r}}_{\mathrm{i} \Theta}\left(\tilde{\mathrm{r}}_{1} \tilde{\mathrm{r}}_{2}\right.$
$\left.\tilde{\mathrm{r}}_{\mathrm{n}}\right)^{-1}$
$\oplus \oplus \oplus$

Step 7: Defuzzy the fuzzy numbers for determining the degree of importance of criteria

Step 8: Creating fuzzy decision matrix

$$
\widetilde{W}=\left(\begin{array}{c}
\widetilde{w}_{1} \\
\widetilde{w}_{2} \\
\vdots \\
\widetilde{w}_{3}
\end{array}\right)
$$

$$
X=\left|\begin{array}{ccc}
{\left[x_{11}^{a}, x_{11}^{b}, x_{11}^{c}\right]} & {\left[x_{12}^{a}, x_{12}^{b}, x_{12}^{c}\right] \ldots \ldots} & {\left[x_{1 m}^{a}, x_{1 m}^{b}, x_{1 m}^{c}\right]} \\
{\left[x_{21}^{a}, x_{21}^{b}, x_{21}^{c}\right]} & {\left[x_{22}^{a}, x_{22}^{b}, x_{22}^{c}\right] \ldots \ldots} & {\left[x_{2 m}^{a}, x_{2 m}^{b}, x_{2 m}^{c}\right]} \\
\ldots \ldots & \ldots \ldots \\
{\left[x_{n 1}^{a}, x_{n 1}^{b}, x_{n 1}^{c}\right]} & {\left[x_{n 2}^{a}, x_{n 2}^{b}, x_{n 2}^{c}\right] \ldots \ldots} & {\left[x_{n m}^{a}, x_{n m}^{b}, x_{n m}^{c}\right]}
\end{array}\right|
$$

Where $x_{i j}^{a}, x_{i j}^{b}, x_{i j}^{c}$ respectively are the lower, middle and upper values of a

triangular membership function of $\mathrm{i}^{\text {th }}$ alternative with respect to $\mathrm{j}^{\text {th }}$ criterion.

Step 9: Normalizing the fuzzy decision matrix using vector normalization process.

$$
\begin{aligned}
& r_{i j}^{a}=\frac{x_{i j}^{a}}{\sqrt{\sum_{i=1}^{n}\left[\left(x_{i j}^{a}\right)^{2}+\left(x_{i j}^{b}\right)^{2}+\left(x_{i j}^{c}\right)^{2}\right]}} \\
& r_{i j}^{b}=\frac{x_{i j}^{b}}{\sqrt{\sum_{i=1}^{n}\left[\left(x_{i j}^{a}\right)^{2}+\left(x_{i j}^{b}\right)^{2}+\left(x_{i j}^{c}\right)^{2}\right]}}
\end{aligned}
$$


A Study on Job Preferences Given in Job Change Decision of Startup Company Workers in Chennai Using Integrated Fuzzy Multi Criteria Decision Making Methodology

$$
r_{i j}^{c}=\frac{x_{i j}^{c}}{\sqrt{\sum_{i=1}^{n}\left[\left(x_{i j}^{a}\right)^{2}+\left(x_{i j}^{b}\right)^{2}+\left(x_{i j}^{c}\right)^{2}\right]}}
$$

Step 10: Weighted normalized fuzzy decision matrix

$$
\begin{aligned}
& v_{i j}^{a}=w_{j} r_{i j}^{a} \\
& v_{i j}^{b}=w_{j} r_{i j}^{b} \\
& v_{i j}^{c}=w_{j} r_{i j}^{c}
\end{aligned}
$$

Step 11: Overall ratings of beneficial and non-beneficial criteria for each alternative is calculated, the overall ratings of an alternative for lower, middle and upper values of membership function.

For beneficial criteria, $S_{I}^{+a}=\sum_{j=1}^{m} v_{i j}^{a}\left(j \in J^{\max }\right)$

$$
\begin{aligned}
& S_{I}^{+b}=\sum_{j=1}^{m} v_{i j}^{b}\left(j \in J^{\text {max }}\right) \\
& S_{I}^{+c}=\sum_{j=1}^{m} v_{i j}^{c}\left(j \in J^{\text {max }}\right)
\end{aligned}
$$

For non-beneficial criteria, $S_{I}^{-a}=\sum_{j=1}^{m} v_{i j}^{a}\left(j \in J^{\text {min }}\right)$

$$
\begin{aligned}
& S_{I}^{-b}=\sum_{j=1}^{m} v_{i j}^{b}\left(j \in J^{m i n}\right) \\
& S_{I}^{-c}=\sum_{j=1}^{m} v_{i j}^{c}\left(j \in J^{m i n}\right)
\end{aligned}
$$

Step 12: Overall performance index $\left(\mathrm{S}_{\mathrm{i}}\right)$, defuzzified values are calculated using the vertex method

$S_{i}\left(S_{i}^{+}, S_{i}^{-}\right)=\sqrt{\frac{1}{3}\left[\left(S_{i}^{+a}-S_{i}^{-a}\right)^{2}+\left(S_{i}^{+b}-S_{i}^{-b}\right)^{2}+\left(S_{i}^{+c}-S_{i}^{-c}\right)^{2}\right]}$

Step 13: The alternative with the highest overall performance index is the best choice.

\section{Case study}

Dissatisfaction in job leads to unhappiness of the worker that affect one's performance and social life. Thus it is important for individuals to carefully evaluate the job alternatives based on factors considered to be important to them. Job change decision is defined as a multiple criteria MCDM problem. In this paper dealt with the job change difficulties of start-up company workers in Chennai. Start-up companies workers mostly hangout due to dissatisfied salary and job nature. Many even get confused what job is suitable for them and are in an urge to prefer the right career. The job preferences of workers are studied using hybrid approach as a methodology integrating FAHP and FMOORA. The criteria and alternatives to define a job change decision are created using a simple questionnaire. The questionnaire answered by the experts helped in defining the following job change criteria and job change alternatives. The criteria are Salary (C1), Work location (C2), Self-respect and identity (C3), Work environment (C4), Effects on 
R. Sophia Porchelvi and B. Snekaa

family (C5), Job nature and security (C6), Career growth (C7), Vested benefits (C8).The alternatives are Working in the same company with a change of job role (A1), IT companies (A2), Product based companies (A3), Government jobs (A4), Change of field (Banking, Teaching etc.) (A5), Entrepreneurship (A6).

\begin{tabular}{ll}
\hline Linguistic variables & $\begin{array}{l}\text { Trapezoidal fuzzy numeric } \\
\text { expressions }\end{array}$ \\
\hline Equal importance (E.I) & $(1,1,1,1)$ \\
\hline Moderate importance (M.I) & $(1,2,4,5)$ \\
\hline Strong importance (S.I) & $(3,4,6,7)$ \\
Very strong importance (V.S.I) & $(5,6,8,9)$ \\
Extreme importance (E.I) & $(7,8,9,9)$ \\
\hline
\end{tabular}

Table 1: Linguistic variables for decision criteria and trapezoidal fuzzy numeric expressions

Fuzzy AHP technique is used to determine the decision hierarchy of criteria and alternatives is designed and is shown in figure 1.
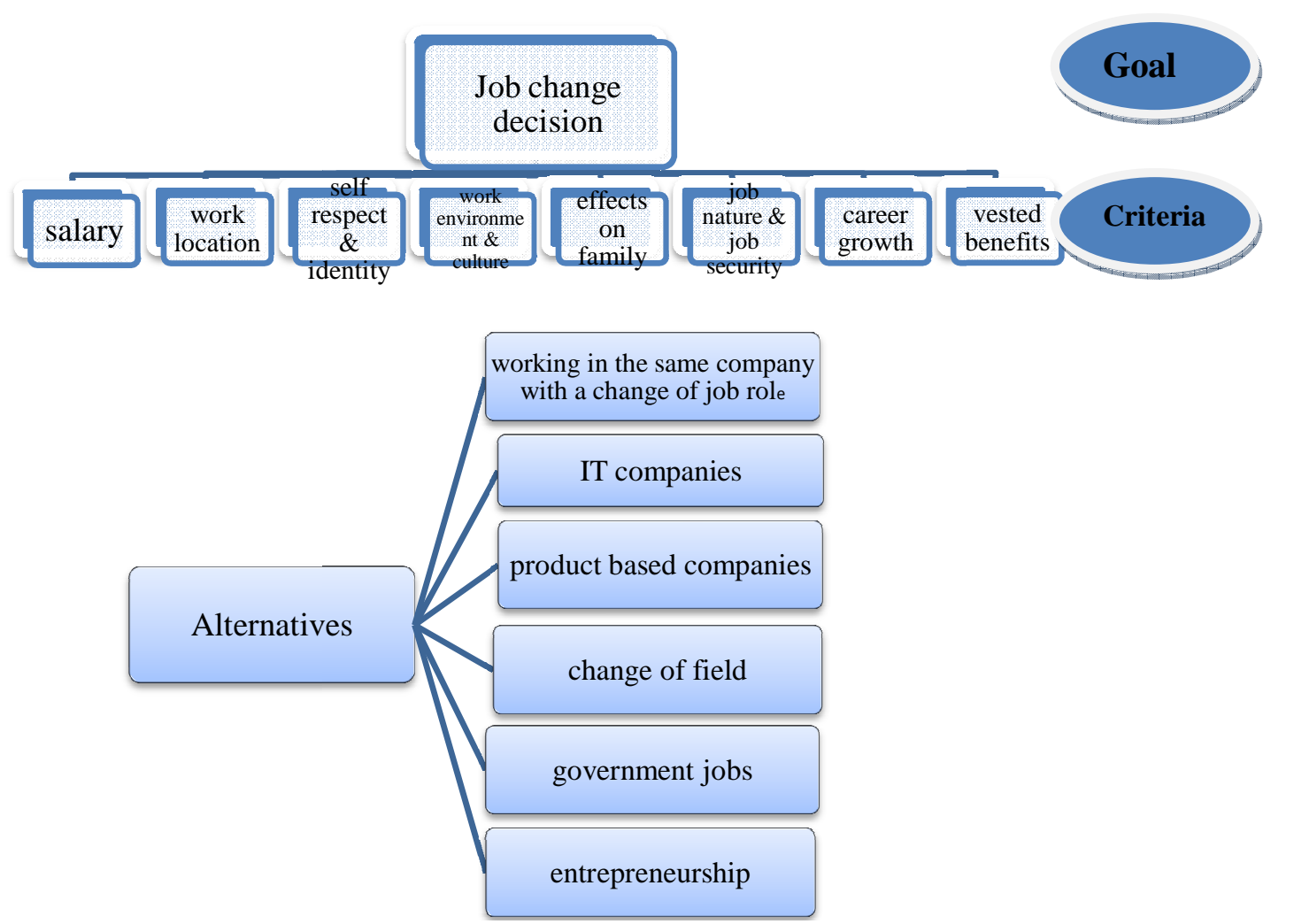

Figure 1: Hierarchical structure of criteria and alternatives 
A Study on Job Preferences Given in Job Change Decision of Startup Company Workers in Chennai Using Integrated Fuzzy Multi Criteria Decision Making Methodology

\begin{tabular}{ll}
\hline Linguistic variables & Trapezoidal fuzzy numeric expressions \\
\hline Very poor (V.P) & $(0,0,1,2)$ \\
Poor (P) & $(1,2,3,4)$ \\
Medium poor (M.P) & $(2,3,4,5)$ \\
Fair (F) & $(4,5,6,7)$ \\
Medium good (M.G) & $(5,6,7,8)$ \\
Good (G) & $(6,7,8,9)$ \\
Very good (V.G) & $(8,9,10,10)$ \\
\hline
\end{tabular}

Table 2: Linguistic variables for alternatives and trapezoidal fuzzy numeric expressions

A questionnaire on pair wise comparison of criteria was created and distributed to experts. Answers in the form of linguistic variables are given numerical expressions and consistent criteria pair wise comparison matrix is created. Then, Geometric mean calculated for each criterion is shown in the table 1 and criteria weight calculated is shown in the table 2.

\begin{tabular}{ccccc}
\hline Geometric mean, $\tilde{\boldsymbol{r}}_{\boldsymbol{i}}$ & $\mathbf{A}$ & $\mathbf{b}$ & $\mathbf{C}$ & $\mathbf{d}$ \\
\hline$\tilde{\boldsymbol{r}}_{\mathbf{1}}$ & 0.7064 & 0.7995 & 1.0273 & 1.2014 \\
$\tilde{\boldsymbol{r}}_{\mathbf{2}}$ & 0.4358 & 0.5308 & 0.7336 & 0.8121 \\
$\tilde{\boldsymbol{r}}_{\mathbf{3}}$ & 4.8687 & 5.5636 & 6.4672 & 6.6830 \\
$\tilde{\boldsymbol{r}}_{\mathbf{4}}$ & 0.6855 & 0.8257 & 1.0925 & 1.2517 \\
$\tilde{\boldsymbol{r}}_{\mathbf{5}}$ & 1.4534 & 1.6983 & 2.1996 & 2.4741 \\
$\tilde{\boldsymbol{r}}_{\mathbf{6}}$ & 1.7257 & 2.0516 & 2.6761 & 2.9935 \\
$\tilde{\boldsymbol{r}}_{\mathbf{7}}$ & 2.4462 & 2.7929 & 2.8809 & 3.5255 \\
$\tilde{\boldsymbol{r}}_{\mathbf{8}}$ & 1.0390 & 1.2309 & 1.5894 & 1.8046
\end{tabular}

Table 3

\begin{tabular}{ccccc}
\hline Criteria weights, $\widetilde{\boldsymbol{w}}_{\boldsymbol{i}}$ & $\mathrm{A}$ & $\mathbf{B}$ & $\mathbf{C}$ & $\mathbf{d}$ \\
\hline$\widetilde{\boldsymbol{w}}_{\mathbf{1}}$ & 0.0340 & 0.0429 & 0.0663 & 0.0899 \\
$\widetilde{\boldsymbol{w}}_{\mathbf{2}}$ & 0.0210 & 0.0285 & 0.0473 & 0.0607 \\
$\widetilde{\boldsymbol{w}}_{\mathbf{3}}$ & 0.2347 & 0.2982 & 0.4171 & 0.4999 \\
$\widetilde{\boldsymbol{w}}_{\mathbf{4}}$ & 0.0330 & 0.0443 & 0.0705 & 0.0936 \\
$\widetilde{\boldsymbol{w}}_{\mathbf{5}}$ & 0.0701 & 0.0910 & 0.1419 & 0.1851 \\
$\widetilde{\boldsymbol{w}}_{\mathbf{6}}$ & 0.0832 & 0.1100 & 0.1726 & 0.2239 \\
$\widetilde{\boldsymbol{w}}_{\mathbf{7}}$ & 0.1179 & 0.1497 & 0.1858 & 0.2637 \\
$\widetilde{\boldsymbol{w}}_{\mathbf{8}}$ & 0.0501 & 0.0660 & 0.1025 & 0.1350 \\
\hline
\end{tabular}

Table 4 


\section{R. Sophia Porchelvi and B. Snekaa}

Best non-fuzzy performance value (BNP) is obtained from defuzzifying fuzzy criteria weights using centre of area COA. For trapezoidal number COA is given as

$$
C O A=\frac{a+b+c+d}{4}
$$

\begin{tabular}{cccc}
\hline Criteria & Name of the criteria & Weight & Rank \\
\hline C1 & Salary & 0.0583 & 7 \\
C2 & Work location & 0.0394 & 8 \\
C3 & Self-respect and identity & 0.3625 & 1 \\
C4 & Work environment and culture & 0.0604 & 6 \\
C5 & Effects on family & 0.1220 & 4 \\
C6 & Job nature and security & 0.1474 & 3 \\
C7 & Career growth & 0.1793 & 2 \\
C8 & Vested benefits & 0.0884 & 5 \\
\hline
\end{tabular}

Table 5: Importance measures of criteria

From the importance measures of criteria it is found criteria weightage ranges in the order c3 $>c 7>c 6>c 5>c 8>c 4>c 1>c 2$. Fuzzy MOORA approach is used to determine the alternatives after the weights of the criteria have been found by fuzzy AHP method. Fuzzy decision matrix on job alternatives is formed with the opinion of the experts. The fuzzy decision matrix is then normalized by vector normalization process by using 6.2. After normalization weighted normalized fuzzy decision matrix is obtained by multiplying the normalized fuzzy criteria values with the corresponding values of crisp criteria weights by using 6.3 . The vertex method is used to defuzzify the overall ratings for both beneficial and non-beneficial attributes by using 6.4and the values of overall performance index is calculated by using 6.5 and the final ranking of alternatives are calculated.

\begin{tabular}{cccc}
\hline Alternative & Name of the alternative & $\begin{array}{c}\text { Performance index } \\
\left(\boldsymbol{S}_{\boldsymbol{i}}\right)\end{array}$ & Rank \\
\hline A1 & Working in the same company & 0.124 & 5 \\
A2 & IT companies & 0.126 & 4 \\
A3 & Product based companies & 0.128 & 3 \\
A4 & Government jobs & 0.158 & 1 \\
A5 & Change of field & 0.112 & 6 \\
\hline A6 & Entrepreneurship & 0.144 & 2 \\
\hline
\end{tabular}

Table 6: Ranking of alternatives based on overall performance index $\left(S_{i}\right)$

It is observed from the table 4 that the highest performance index occurs in the order A 4 $>$ A $5>A$ 3 $>$ A 2 $>$ A $1>A 6$ that shows Government jobs stands first in job change decision followed by entrepreneurship. 
A Study on Job Preferences Given in Job Change Decision of Startup Company Workers in Chennai Using Integrated Fuzzy Multi Criteria Decision Making Methodology

\section{Conclusion}

The job preferences of workers are studied using hybrid approach as a methodology integrating fuzzy analytic hierarchy process and fuzzy MOORA. It was found using FAHP that individuals gave weightage to criteria self-respect \& identity followed by career growth. And using FMOORA analysis over job alternatives shows individual's interest over Government jobs followed by entrepreneurship. One of the most important decision making in one's life is job decision making, it should be optimistically approached. Fuzzy multi criteria decision making methods has helped the workers with best alternatives and the problem of which type of job field the individuals has to choose for the betterment of his/her career has been discussed successfully in this paper.

Acknowledgment. We would like to thank reviewers for their insightful comments on our paper, as these comments led us to an improvement of our work.

\section{REFERENCES}

1. L.A.Zadeh, Fuzzy sets, Information and Control, 8(3) (1965) 338-353.

2. G.J.Klir and B.Yan, Fuzzy Sets and Fuzzy Logic Theory and Applications, PrenticeHall International, Inc. London, 1995.

3. C.Loganathan and V.Pushpalatha, Circulant interval valued fuzzy matrices, Annals of Pure and Applied Mathematics, 16(2) (2018) 313-322.

4. R.Pradhan and M.Pal, Intuitionistic fuzzy linear transformations, Annals of Pure and Applied Mathematics, 1(1) (2012) 57-68.

5. R.E.Bellman and L.A.Zadeh, Decision-making in a fuzzy environment, Management Science, 17(4) (1970) 141-164.

6. S.J.Chen, C.L.Hwang and F.P.Hwang, Fuzzy Multiple Attribute Decision Making, Springer-Verlag, Berlin, 1992.

7. C.L.Hwang and K.Yoon, Multiple Attribute Decision Making methods and Applications, Springer, Berlin, Germany, 1981.

8. T.L.Saaty, The Analytic Hierarchy Process, McGraw-Hill, New York, 1980.

9. J.J.Buckley, Fuzzy hierarchical analysis, Fuzzy Sets and Systems, 17(3) (1985) 233247.

10. P.J.M.Van Laarhoven and W.Pedrycz, A fuzzy extension of Saaty's priority theory, Fuzzy Sets and Systems, 11(3) (1983) 229-241.

11. D.Y.Chang, Applications of the extent analysis method on fuzzy AHP, European Journal of Operational Research, 95 (1996) 649-655.

12. A.Balezantis, T.Balezantis and W.K.M.Brauers, Multimoora-fg: a multi-objective decision making method for linguistic reasoning with an application to personnel selection, Informatica, 23(2) (2012) 173-190.

13. W.K.M.Brauers and E.K.Zavadskas, The MOORA method and its application to privatization in a transition economy, Control and Cybernetics, 35(2) (2006) 445469.

14. Gokay Akkaya, Betul Turanoglu and Sinan Oztas, An integrated fuzzy AHP and fuzzy MOORA approach to the problem of industrial engineering sector choosing, Ataturk University, Erzurum 25240, Turkey. 


\section{R. Sophia Porchelvi and B. Snekaa}

15. S.Alp and T.K.Ozkan, Job choice with multi-criteria decision making approach in a fuzzy environment, International Review of Management and Marketing, 5(3) (2015) 165-172.

16. S.Elizabeth and L.Sujatha, Medical diagnosis based on interval valued fuzzy number matrices, Annals of Pure and Applied Mathematics, 7(1) (2014) 91-96. 